\title{
Vino, fibrinolisis y salud
}

\author{
Consuelo Pasten, Hernán G renett \\ Wine, fibrinolysis and health
}

\begin{abstract}
Cardiovascular diseases are the leading cause of death in both men and women in the world. Epidemiological and experimental studies have associated moderate wine consumption (1 to 2 glasses/day) with a decrease in cardiovascular diseases. This decrease is probably due to the effect of ethanol and polyphenols present in the wine. The cardioprotective benefit of wine may be due, in part, to a modulation of the expression of proteins involved in fibrinolysis. Endothelial cells (ECs) play a major role in maintaining normal hemostasis, regulating the balance between the synthesis and interaction of proteins that promote clot formation (thrombosis) and fibrinolytic proteins that facilitate clot lysis. These cells are a major site of synthesis of fibrinolytic proteins, such as tissue type plasminogen activator (t-PA), urokinase-type plasminogen activator (u-PA) and the major inhibitor/regulator of fibrinolysis, PAI-1. EC-mediated fibrinolysis is regulated and localized to the EC surface through specific receptors for $\mathrm{u}-\mathrm{PA}, \mathrm{t}-\mathrm{PA}$ and plasminogen. Evidence indicates that ethanol and polyphenols present in wine increase EC localized fibrinolisis. Upregulation of t-PA and u-PA activity and downregulation of PAI-1 may account, at least in part, for this net increase in fibrinolytic activity. The purpose of this review is to cover the main molecular and physiological aspects of moderate wine consumption mediated increase in fibrinolysis and reduction in cardiovascular risk (Rev Méd Chile. 2006; 134: 1040-8).
\end{abstract}

(Key w ords: Cardiovascular diseases; Fibrinolysis; Thrombosis; Wine)

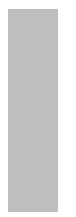

Recibido el 28 de junio, 2005. Aceptado el 14 de marzo, 2006.

Este estudio fue financiado por: The National Heart, Lung, and Blood Institute (NHLBI) Program Project Grant PPG HL70610 «Mechanism of alcohol and Polyphenols Cardioprotection» Project 2.

Division of Cardiovascular Disease, Department of Medicine, University of Alabama at Birmingham, Alabama, Estados Unidos de Norteamérica.

A pesar de muchos años de investigaciones epidemiológicas y experimentales, aún no se ha determinado la causa precisa de las enfermedades cardiovasculares, principalmente debido a

Correspondencia a: Hernán E. Grenett, Ph. D. Universidad de Alabama, Birmingham. 84519 Th. Av. South, Bevil Biomedical Research Building, 830, Birmingham, Al 35294. Estados Unidos. Tel.: 205-975-5251. Fax: 205-975-7121. E mail: hgrenett@cardio.dom.uab.edu que su origen no se puede atribuir a una sola causa. Se puede hablar de factores de riesgo, refiriéndose a un grupo de anomalías y condiciones personales que desencadenan su desarrollo. Entre ellos, tenemos factores individuales, tales como el sexo, edad, antecedentes familiares o genéticos, los cuales no son modificables. Factores de riesgo directos incluyen: niveles elevados de colesterol total y lipoproteínas de baja densidad (LDL), bajos niveles de lipoproteínas de alta densidad (HDL), tabaquismo, hipertensión, diabetes y una dieta no saludable. Entre los factores 
indirectos se encuentra el sedentarismo, la obesidad y el estrés.

Las enfermedades cardiovasculares pueden ser divididas en dos importantes grupos. Complicaciones de tipo vascular, como hipertensión o derrames y aquellas que afectan directamente al miocardio, por ejemplo cardiomiopatías o arritmias. En ambos grupos, están involucrados mecanismos celulares y moleculares, que se alteran en respuesta a interacciones con factores sistémicos 0 medioambientales. Debido a que actualmente son consideradas una de las primeras causas de muerte en el ámbito mundial, es de vital importancia clarificar sus orígenes, evolución, prevención, y es absolutamente necesario estudiar nuevos compuestos que produzcan una acción directa sobre estos mecanismos. Así, factores sistémicos que puedan inhibir o minimizar estas disfunciones, podrían tener un efecto directo sobre su desarrollo ${ }^{1}$.

\section{VINO Y FUNCIÓN CARDIOVASCULAR}

Estudios desarrollados hace más de 20 años pusieron de manifiesto que los países de la cuenca mediterránea europea (España, Italia, Francia, Grecia y Portugal), tenían un menor porcentaje de infartos al miocardio y una menor tasa de mortalidad por cáncer que la población mundial. En busca de las causas de esta situación, se llegó a la conclusión que la dieta tenía un papel fundamental. La llamada «dieta mediterránea» es un importante factor a considerar cuando se trata de prevención de las enfermedades cardiovasculares. Esta dieta se basa en la ingesta de aceite de oliva, frutas, verduras, pastas, arroz, legumbres, pescado, pan integral y un consumo moderado de vino $^{2}$.

Un ejemplo específico es la llamada sparadoja francesa». Francia es un país caracterizado por consumir en su dieta una alta cantidad de grasas saturadas. A raíz de esto, se esperaría una alta tasa de mortalidad por enfermedades cardiovasculares. Sin embargo, las estadísticas indican que tiene $40 \%$ menos mortalidad por esta causa, que otros países industrializados. Más aún, un gran porcentaje de la población adulta consume alcohol en forma regular, lo cual, sumado a la dieta alta en grasas saturadas, elevaría los niveles de triglicéri- dos en el plasma. No obstante ello, esta situación no se refleja en un alto riesgo de enfermedades cardiovasculares. Se cree que el consumo moderado de alcohol, en lugar de producir un efecto dañino, invierte la situación, produciendo una acción cardioprotectora ${ }^{2,3}$.

El balance normal del organismo es mantenido por una interacción compleja y regulada de varios constituyentes del sistema hemostático, entre ellos, proteínas fibrinolíticas, compuestos que permiten la coagulación y plaquetas circulantes ${ }^{4}$. La formación de un trombo comienza con la agregación de las plaquetas, las que interactúan con proteínas del plasma, permitiendo la polimerización del fibrinógeno por acción de la trombina. La disolución natural de este coágulo o trombo se logra por la acción conjunta de un sistema que incluye enzimas proteolíticas, activadores específicos e inhibidores, tanto de las proteasas como de sus activadores, los que incluyen los fragmentos activados del factor de Hageman, el activador tisular del plasminógeno (t-PA) y el activador del plasminógeno tipo urocinasa (u$\mathrm{PA})^{3,5,6}$. Estos activadores convierten al cimógeno en plasmina, forma activa del plasminógeno y que es, en última instancia, la proteasa responsable de la disolución del trombo.

La regulación del proceso de fibrinolisis comienza con la liberación de los activadores del plasminógeno (t-PA y u-PA) desde las células endoteliales, los cuales se unen a sus respectivos receptores en la superficie celular. Del mismo modo, el plasminógeno circulante se une a sus receptores en la superficie endotelial y es activado por la acción de t-PA y u-PA, generando la forma activa del plasminógeno, plasmina. La duración y magnitud de la fibrinolisis está regulada por el inhibidor circulante de la plasmina, el alfa-2 antiplasmina y por el inhibidor de los activadores del plasminógeno, PAI-13,5,6. (Figura 1A). Las plaquetas también contribuyen a mantener la integridad del tapón hemostático, liberando PAI-1 en los lugares de lesión vascular, una vez que se ha producido la agregación plaquetaria ${ }^{5-7}$.

El consumo moderado de vino y su relación con patologías cardiovasculares, ha sido ampliamente estudiado. A pesar de que existe consenso general que el consumo excesivo de alcohol ( $40 \mathrm{~g}$ o 4 vasos al día) aumenta la mortalidad por cirrosis y algunos tipos de cánceres, su consumo 


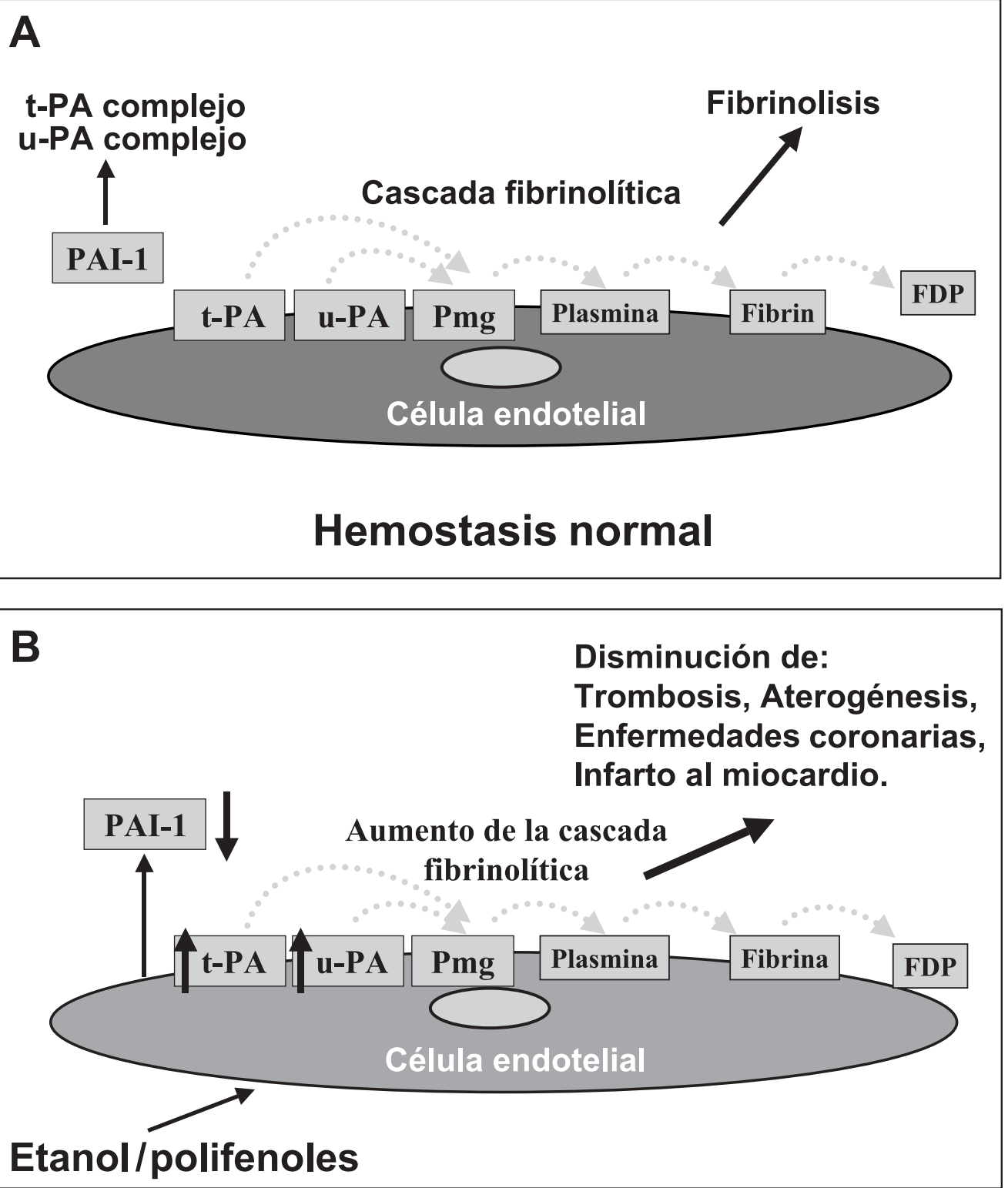

Figura 1. Ilustración esquemática del proceso de fibrinolisis. A. En condiciones normales, las células endoteliales producen t-PA, u-PA y PAI-1, el inhibidor de la fibrinolisis. Tanto t-PA como u-PA, activan el plasminógeno (Pmg) generando plasmina. La plasmina activada actúa sobre la fibrina, disolviendo el coágulo o trombo, produciendo fibrinolisis y generando los productos de degradación de fibrina (FDP). PAI-1 regula el proceso, neutralizando el exceso de t-PA, u-PA en la superficie de las células endoteliales. B. El etanol y los polifenoles del vino alteran la actividad, interacción y expresión de t-PA, u-PA y PAI-1. Esta alteración produce un aumento en la actividad fibrinolítica de superficie (generación de plasmina), y por lo tanto, aumenta la degradación de fibrina a productos de degradación de fibrina. Así, el consumo moderado de vino puede aumentar y mantener en forma sostenida la actividad fibrinolítica de superficie y reducir el niesgo de trombosis, aterogénesis, enfermedades cardiovasculares e infarto cardíaco. 
con moderación (1-2 vasos al día) tiene múltiples efectos bioquímicos y celulares benéficos para el sistema cardiovascular ${ }^{4}$. Diversos estudios demuestran que existe una relación entre consumo de alcohol y riesgo de enfermedades cardiovasculares. Se ha podido establecer que quienes beben en forma moderada tienen menor riesgo de padecer problemas cardiovasculares comparados con aquellos bebedores excesivos ${ }^{8,9}$.

Estudios epidemiológicos y experimentales, muestran que el alcohol induce cambios en el perfil lipídico y en la función plaquetaria. Además, se ha observado cambios vasculares a nivel del funcionamiento del miocardio, los que reducirían el riesgo de padecer enfermedades cardiovasculares ${ }^{4}$.

Por ejemplo, se ha demostrado que el alcohol aumenta los niveles del llamado «colesterol bueno» o HDL (lipoproteínas de alta densidad), lo cual se relaciona directamente con un bajo riesgo de arteriosclerosis e infarto al miocardio ${ }^{10}$. En el ámbito de plaquetas, estudios desarrollados in vivo, utilizando como modelos ratas, conejos y perros, han demostrado que el consumo moderado de alcohol inhibe la activación plaquetaria y formación de trombos ${ }^{11-13}$. Estudios in vitro, utilizando concentraciones bajas de etanol (25 $\mathrm{mM})$, la inhibición de la agregación y secreción plaquetaria involucraría la inhibición de la fosfolipasa $\mathrm{A}^{14,15}$.

Con relación a la coagulación, existiría una asociación entre el consumo moderado de alcohol y la disminución de los niveles de fibrinógeno en el plasma ${ }^{16}$. Este efecto podría ser atribuido a su acción antiinflamatoria. Otro estudio demostró que bajas dosis de etanol redujeron los niveles de fibrinógeno en $18-20 \%$ en experimentos tanto in vivo como in vitro ${ }^{17}$.

En conclusión, estudios epidemiológicos han demostrado que individuos que consumen alcohol en forma moderada, tienen un menor riesgo de enfermedad cardiovascular ${ }^{18,19}$. Los mecanismos moleculares que permitirían esta acción, no están del todo claros. La mayoría de las investigaciones se han focalizado sobre el hecho de que el alcohol induce cambios favorables en los niveles de lípidos circulantes ${ }^{20}$. Por otra parte, estudios experimentales han sugerido que el efecto benéfico del etanol podría deberse a su capacidad de estimular la fibrinolisis a través de cambios en la actividad, niveles o interacción de uno o más componentes del sistema fibrinolítico, tales como t-PA, u-PA, PAI-1 y fibrinógeno ${ }^{18,16}$.

\section{AlCOHOL Y FIBRINOLSIS}

Como se mencionó anteriormente, PAI-1 desempeña una importante función en la fibrinolisis mediante la inhibición de t-PA y u-PA y, por lo tanto, en la disolución del coágulo de fibrina. PAI1 se expresa en muchos tipos celulares, incluyendo células endoteliales ${ }^{21}$, células del músculo del corazón $^{22}$ y hepatocitos ${ }^{23}$. Su expresión es estimulada por una variedad de agentes tales como: citocinas inflamatorias ${ }^{24}$, trombina ${ }^{25}$ lipoproteínas de muy baja densidad (VLDL) ${ }^{19}$ e insulina 0 proinsulina ${ }^{26}$. El PAI-1 circulante tiene una vida media de 90 min. La vitronectina, una proteína abundante en el plasma, estabiliza al PAI-1 en la forma activa y prolonga su vida media a 120 $\min ^{27}$.

Meade y cols ${ }^{28}$ fueron los primeros en demostrar una relación entre el alcohol y fibrinolisis, observando un aumento en la actividad del PAI-1 y una disminución en la actividad del antígeno tPA una hora después del consumo de alcohol durante la cena. Sin embargo, durante la noche, la actividad del PAI-1 retornaba a los niveles basales, mientras que los niveles de t-PA permanecían altos, produciendo un aumento en la actividad de t-PA circulante a la mañana siguiente. En estudios similares, se ha demostrado que bebedores moderados tienen significativamente más baja incidencia de eventos cardiovasculares y presentar niveles de t-PA en el plasma más altos que los no bebedores ${ }^{29}$. Incluso, en sujetos que sufren de alcoholismo crónico, pero bajo tratamiento de abstinencia, se ha observado un aumento en la fibrinolisis, principalmente debido a la disminución de PAI-130.

Estudios realizados in vitro, han demostrado que una dosis baja de etanol $(0,01-0,10 \%, v / v)$ aumenta la actividad fibrinolítica, la cual va acompañada de un aumento en la expresión del tPA y u-PA ${ }^{31-33}$.

También se ha visto que el etanol regula la expresión de una variedad de genes que incluyen, la piruvato descarboxilasa ${ }^{34}$, la GRP78 proteína inducida por estrés ${ }^{35}$, el gen traqueobranquial 
mucino ${ }^{36}$ y el gen para la citocromo $\mathrm{P} 4502^{37}$. Grenett y $\operatorname{cols}^{38}$ han demostrado in vitro, en células aisladas de cordón umbilical humano (HUVEC), que el etanol disminuye la expresión de PAI-1 y que esta represión es a nivel transcripcional. Recientemente hemos demostrado que existen factores de transcripción que se unirían a una región promotora del gen del PAI-1. En experimentos in vitro, usando HUVECs, hemos identificado un fragmento de 251 pares de bases en el promotor del PAI-1 que responde al etanol ${ }^{39}$. Una búsqueda computacional en el banco de genes (Gen Bank) ha identificado numerosos factores de transcripción que podrían ser los responsables de la represión del PAI-1 por el etanol ${ }^{35-37,40,41}$. Así, una disminución en la expresión del PAI-1, se traduciría en un aumento neto en la actividad fibrinolítica en la superficie de las células endoteliales.

\section{POLFENOLES Y FIBRINOLISIS}

Las evidencias experimentales demuestran que consumir en forma regular y moderada vino, disminuye el riesgo de padecer complicaciones cardiovasculares. El vino, además de alcohol, contiene una gran variedad de compuestos polifenólicos que actuarían como antioxidantes. Estudios realizados con vino desalcoholizado han demostrado las propiedades cardioprotectoras de estos polifenoles ${ }^{42-44}$.

Entre los compuestos polifenólicos del vino se encuentran los ácidos fenólicos (ácidos ferúlicos, cumáricos, cinámicos, vainíllicos, cafeicos); trihidroxistilbenos (resveratrol y polidatina) y flavonoides (catequinas, epiquercetina, quercetina) ${ }^{45}$. El contenido de estos compuestos es menor en el vino blanco que en el tinto. Las diferencias se deben básicamente a la variedad vinífera, al rendimiento de la cosecha y a las condiciones climáticas y técnicas aplicadas a cada procesamiento. El contenido medio de compuestos polifenólicos en un vino blanco es del orden de 150 a $250 \mathrm{mg} / \mathrm{L}$, mientras que en el vino tinto oscila entre 1.000 y $4.000 \mathrm{mg} / \mathrm{L}^{46}$ (Tabla 1 ).

En patologías cardiovasculares, se ha demostrado que los compuestos polifenólicos del vino tienen variadas funciones: disminuyen la oxidación de las LDL (causantes de la ateroesclerosis); inhiben la agregación plaquetaria ${ }^{14,15}$; aumentan la óxido nítrico sintasa y la vasorrelajación del endotelio ${ }^{4}$; mejoran la vascularidad y disminuyen el daño oxidativo al $\mathrm{ADN}^{7}$; y aumentan significati-

Tabla 1. N iveles de compuestos fenólicos en vino blanco y tinto

\begin{tabular}{|lrr|}
\hline & Blanco & Tinto \\
\hline Flavonoides & & \\
$\quad$ Flavonoles monoméricos (por ejemplo: catequina) & 15 & 100 \\
Proantocianidina y taninos condensados & 25 & 1.000 \\
Flavonoles (por ejemplo: quercetina) & $\mathrm{n} / \mathrm{a}$ & 100 \\
Total mg/L & 40 & 1.365 \\
& & \\
No flavonoides & 130 & 60 \\
Derivados de cinamatos & 15 & 60 \\
Derivados de benzenos & 100 & 250 \\
Taninos hidrolizables & 0,5 & 7 \\
Estilbenos (por ejemplo: resveratrol) & 245,5 & 377 \\
Total mg/L & & 1,742 \\
Total fenoles mg/L & 285,5 & \\
\hline
\end{tabular}

Adaptado con permiso de Cordova y cols. The cardiovascular protective effect of red wine. J Am Coll Surg 2005; 3: 428-439. 
vamente la fibrinolisis ${ }^{31}$. Recientemente, se ha reportado que quercetina inhibe la proliferación en cultivos de células endoteliales de origen microvascular ${ }^{47}$ y la expresión de citocinas proinflamatorias en macrófagos estimulados con LPS (lipopolisacáridos) ${ }^{48}$.

Nueva e importante característica de estos compuestos polifenólicos, es la de ejercer efectos sobre la expresión de ciertos genes y que esta regulación es a nivel transcripcional. Se ha demostrado que el resveratrol inhibe la expresión de la cicloxigenasa-2 y del factor tisular ${ }^{49,50}$. Acido gálico, inhibe la translocación nuclear del NF-кB (factor nuclear kappaB) y aumenta la expresión de genes que codifican para VCAM-1, ICAM-1 (moléculas 1 de adhesión vascular e intercelular, respectivamente) y E-selectina ${ }^{51}$. Estudios in vitro sobre efecto de los polifenoles en la fibrinolisis, han demostrado que catequina, quercetina, epiquercetina y resveratrol aumentan la actividad fibrinolítica en células endoteliales. Esta respuesta podría deberse, en parte, al aumento en la expresión de t-PA y u-PA ${ }^{52}$.

Los polifenoles, entre otras funciones, activan la señalización celular dependiente de óxido nitroso (NO), la cual es modulada por $\mathrm{O}_{2}$ - y la superóxido dismutasa, una enzima que cataliza la formación del peróxido de hidrógeno y oxígeno molecular ${ }^{53}$. Se ha demostrado que la quercetina inhibe la oxidación catalizada por cobre de LDL y permite la eliminación de radicales libres ${ }^{53}$. Recientemente ha sido comunicada una relación entre la concentración de polifenoles del vino y su capacidad vasodilatadora en anillos de aorta de ratas $^{54}$. En experimentos de hibridación in situ, hemos demostrado que polifenoles disminuyen la

\section{REFERENCIAS}

1. Michalsen A, Dobos GJ. Effects of nutritional factors on haemostasis. Hamostaseologie 2005; 1: 13-7.

2. De Lorgeril M, Salen P, Pailuard F, Laporte F, BOucher F, De LeIRIS J. Mediterranean diet and the French paradox: two distinct biogeographic concepts for one consolidated scientific theory on the role of nutrition in coronary heart disease. Cardiovas Res 2002; 3: 503-15. expresión de PAI-1 in vivo en el endotelio aórtico de la rata ${ }^{55}$.

Las evidencias experimentales sugieren que el mecanismo de acción de los polifenoles sería a través de la activación de las proteíno-kinasas activadas por mitógeno (MAPKs). MAPKs son un grupo de proteínas citoplasmáticas que son fosforiladas y que, a su vez, fosforilan a otras proteínas que se encargan de llevar la información biológica desde la membrana celular hasta el núcleo. Se ha demostrado que quercetin y otros polifenoles modulan la actividad de algunas kinasas ${ }^{56}$. Kobuchi y cols, han demostrado que la quercetina regula la expresión de ICAM-1 en células endoteliales humanas, mediante la inhibición de proteína kinasa c-JUN (JNK) ${ }^{57}$. Otros estudios han observado que la quercetina inhibe la expresión de MMP1, proteína involucrada en la desestabilización de la placa ateroesclerótica, bloqueando la proteíno kinasa ERK ${ }^{58}$. Tomando en cuenta estas observaciones, es posible especular que la acción de los polifenoles esté relacionada con la activación 0 modulación de la vía de las MAPKs. En estudios preliminares en nuestro laboratorio, se ha observado que quercetina y catequina regulan la expresión de PAI-1 a través de la MAPKs, JNK y ERK1/2.

En resumen, factores como el etanol y polifenoles que modulan la expresión de proteínas fibrinolíticas, deberían aumentar la fibrinolisis y disminuir trombosis. El entendimiento del mecanismo de acción del vino y de específicos polifenoles en los beneficios para la salud, podría ser una gran contribución a la sociedad. Por el momento, las evidencias nos dicen que un consumo moderado de vino (1 ó 2 vasos por día) puede bajar el riesgo de sufrir enfermedades cardiovasculares.

3. Booyse FM, Aikens ML, Grenett HE. Endothelial cell fibrinolysis: transcriptional regulation of fibrinolytic protein gene expression ( $\mathrm{t}-\mathrm{PA}, \mathrm{u}-\mathrm{PA}$, and PAI-1) by low alcohol. Alcohol Clin Exp Res 1999; 23: 1119-24.

4. Booyse FM, PARKs DA. Moderate wine and alcohol consumption: beneficial effects on cardiovascular disease. Thromb Haemost 2001; 86: 517-28.

5. Handin R, Loscaizo J. Hemostasis, Thrombosis, Fibrinolysis and Cardiovascular disease. En: Braunwald E, ed. Heart Disease. A textbook of 
cardiovascular medicine. PA W.B. Saunders Company, 1988; 1758-9.

6. BOdARY PF, Wickenheiser KJ, EITZMAN DT. Recent advances in understanding endogenous fibrinolysis: implications for molecular-based treatment of vascular disorders. Expert Rev Mol Med 2002; 26: 1-10.

7. Leigthon F, Casanegra P, Maiz A. Alcohol, vino y factores de riesgo cardiovascular. Boletín Ciencia, Vino y Salud, Pontificia Universidad Católica de Chile, 2000; 4: 1-8.

8. Fagrell B, De Faire U, Bondy S, Criqui M, Gaziano M, Gronbaek M et al. The effects of light to moderate drinking on cardiovascular diseases. J Intern Med 1999; 246: 331-40.

9. Klatsky AL, Armstrong MA, Friedman GD. Red wine, white wine, liquor, beer and risk of coronary artery disease hospitalization. Am J Cardiol 1997; 80: 416-20.

10. Hansen AS, Marckmann P, Dragsted LO, Finne Nielsen IL, NieLSEN SE, GronbaeK M. Effect of red wine and red grape extract on blood lipids, haemostatic factors, and other risk factors for cardiovascular disease. Eur J Clin Nutr 2005; 3: 449-55.

11. Baysan O, Kaptan $K$, Erinc $K$, Oztas $Y$, Coskun T, KAYIR H ET AL. Chronic heavy ethanol consumption is associated with decreased platelet aggregation in rats. Tohoku J Exp Med 2005; 2: 8590.

12. Rand ML, Dacosta SM, Chahil A, Kinlough-RathboNE RL. Combined inhibitory effects of ethanol and adenosine on the responses of rabbit platelets to thrombin. Alcohol Clin Exp Res 1995; 5: 1279-85.

13. Demrow HS, Slane PR, Folts JD. Administration of wine and grape juice inhibits in vivo platelet activity and thrombosis in stenosed canine coronary arteries. Circulation 1995; 15: 1182-8.

14. RuBin R. Effect of ethanol on platelet function. Alcohol Clin Exp Res 1999; 6: 1114-8.

15. NguYen A, Packham MA, Rand ML Effects of ethanol on platelet responses associated with adhesion to collagen. Thromb Res 1999; 6: 303-14.

16. HendriKs HF, VAn Der GaAg MS. Alcohol, coagulation and fibrinolysis. Novartis Found Symp 1998; 216: 111-20.

17. WANG Z, Barker TH, FULLeR GM. Alcohol at moderate levels decreases fibrinogen expression in vivo and in vitro. Alcohol Clin Exp Res 1999; 12: 1927-32.
18. De Lange DW, Van De Wiel A. Drink to prevent: review on the cardioprotective mechanisms of alcohol and red wine polyphenols. Semin Vasc Med 2004; 2: 173-86.

19. Mukamal KJ, Jensen MK, Gronbaek M, Stampfer MJ, Manson JE, Pischon T et al. Drinking frequency, mediating biomarkers, and risk of myocardial infarction in women and men. Circulation 2005; 10: 1406-13.

20. Salem RO, Laposata M. Effects of alcohol on hemostasis. Am J Clin Pathol 2005; 123: S96-105.

21. ERIKSSON P, NiLsson L, Karpe F, Hamsten A. Verylow-density lipoprotein response element in the promoter region of the human plasminogen activator inhibitor-1 gene implicated in the impaired fibrinolysis of hypertriglyceridemia. Arterioscler Thromb Vasc Biol 1998; 18: 20-6.

22. Janand-Delenne B, Chagnaud C, Raccah D, Aiessi MC, Juhan-Vague I, Vague P. Fat as a main determinant of plasminogen activator inhibitor 1 level in women. Int J Obes Relat Metab Disord 1998; 22: 312-7.

23. Brown SL, Soberl BE, FuJII S. Attenuation of the synthesis of plasminogen activator inhibitor type 1 by niacin. A potential link between lipid lowering and fibrinolysis. Circulation 1995; 15: 767-72.

24. HaLe M, Berg A, Northoff H, Keul J. Importance of TNF-alpha and leptin in obesity and insulin resistance: a hypothesis on the impact of physical exercise. Exerc Immunol Rev 1998; 4: 77-94.

25. Yamamoto C, Sugato M, Fujinara Y, Kaji T. Selective promotion of plasminogen activator inhibitor1 secretion by activation of proteinase-activated receptor- 1 in cultured human brain microvascular pericytes: comparison with endothelial cells. Biol Pharm Bull 2005; 2: 208-11.

26. Pandolfi A, Iacovielo L, Capani F, Vitacolonna E, Donati MB, Consou A. Glucose and insulin independently reduce the fibrinolytic potential of human vascular smooth muscle cells in culture. Diabetologia 1996; 39: 1425-31.

27. DeLas C, Loskutofr DJ. Historical analysis of PAI-1 from its discovery to its potential role in cell motility and disease. Thromb Haemost 2005; 4: 631-40.

28. Meade TW, Chakrabarti R, Haines AP, North WF, STIRLNG Y. Characteristics affecting fibrinolytic activity and plasma fibrinogen concentrations. $\mathrm{Br}$ Med J 1979; 20: 153-6. 
29. Ridker PM, Vaughan DE, Stampfer MJ, Glynn RJ, HENNEKENS CH. Association of moderate alcohol consumption and plasma concentration of endogenous tissue-type plasminogen activator. JAMA 1994; 28: 929-33.

30. Delahousse B, Mailot F, Gabriel I, Schelenderg F, LAMISSE F, Guel Y. Increased plasma fibrinolysis and tissue-type plasminogen activator/tissue-type plasminogen activator inhibitor ratios after ethanol wiithdrawal in chronic alcoholics. Blood Coagul Fibrinolysis 2001; 1: 59-66.

31. De Gaetano G, Di Castelnuovo A, Donati MB, IACOVIELO L. The mediterranean lecture: wine and thrombosis-from epidemiology to physiology and back. Pathophysiol Haemost Thromb 2003/2004; 33: 466-71.

32. Aikens ML, Benza RL, Grenett HE, Tabengwa EM, Davis GC, Demissie $S$ et aL. Ethanol increased surface-localized fibrinolytic activity in culture endothelial cells. Alcohol Clin Exp Res 1997; 21: 1471-8.

33. Aikens ML, Grenett HE, Benza RL, Tabengwa EM, Davis GC, BoysE FM. Alcohol- induced up regulation of plasminogen activators and fibrinolytic activity in culture human endothelial cells. Alcohol Clin Exp Res 1998; 22: 375-81.

34. LISEN T, HOLENBERg CP, HeinISH JJ. ERA, a novel cisacting element required for autoregulation and ethanol repression of PDC1 transcription in Saccharomyces cerevisae. Mol Microbiol 1996; 21: 621-32.

35. HsieH KP, WiLKe N, HaRRis A, Miles MF. Interaction of ethanol with inducers of glucose-regulated stress proteins. Ethanol potentiates inducers of grp78 transcription. J Biol Chem 1996; 271: 270916.

36. Verma M, Davidson EA. Transcriptional regulation of tracheo-bronchial mucin (TBM) gene by ethanol. Gene 1997; 11: 9-12.

37. Robin MA, Sauvage I, Grandperret T, Descatoire V, Pessayre D, Fromenty B. Ethanol increases mitochondrial cytochrome P450 2E1 in mouse liver and rat hepatocytes. FEBS Lett 2005; 30: 6895-902.

38. Grenett HE, Aikens ML, Tabengwa EM, Davis GC, BooySE FM. Ethanol down regulates transcription of the PAI-1 gene in cultured human endothelial cells. Thromb Res 2000; 15: 247-55.

39. GrenetT HE, Wolkowicz PE, Benza RL, Tresnak JK, WHEELER CG, BOOYSe FM. Identification of a 251bp fragment of the PAI-1 gene promoter that mediates the ethanol-induced suppression of PAI1 expression. Alcohol Clin Exp Res 2001; 25: 62936.

40. Thiele TE, Van DiJiK G, Bernstein IL Ethanolinduced c-Fos expression in rat lines selected for low and high alcohol consumption. Brain Res 1997; 9: 278-82.

41. Mandrekar P, Catalano D, Szabo G. Alcoholinduced regulation of nuclear regulatory factor$\mathrm{kb}$ in human monocytes. Alcohol Clin Exp Res 1997; 21: 988-94.

42. Wang Z, Zou J, Cao K, Hsieh TC, Huang Y, Wu JM. Dealcoholized red wine containing known amounts of resveratrol suppresses atherosclerosis in hypercholesterolemic rabbits without affecting plasma lipid levels. Int J Mol Med 2005; 4: 533-40.

43. Sato M, Ray PS, Maulik G, Maulik N, Engelman RM, BERTEU AA ET AL. Myocardial protection with red wine extract. Journal of Cardiovascular Pharmacology 2000; 35: 263-8.

44. Martínez-Valverde I, Periago MJ, Ros G. Nutritional importance of phenolic compounds in the diet. Arch Latinoam Nutr 2000; 50: 5-18.

45. SOIFAS GJ, Diamandis EP, GoldBerg DM. Wine as a biological fluid-history, production, and role in disease prevention. J Clin Lab Anal 1997; 11: 287-313.

46. Córdova AC, Jackson LS, Berke-Schlessel DW, SumPIO BE. The cardiovascular protective effect of red wine. J Am Coll Surg 2005; 3: 428-39.

47. Fan PS, Gu ZL, Sheng R, Luang ZQ, Wang XX, Zhu $Y$. Inhibitory effect of quercetin on proliferation of human microvascular endothelial cells in vitro. Acta Pharmacol Sin 2003; 24: 1231-4.

48. Cho SY, Park SJ, Kwon MJ, Jeong TS, Boк SH, Choi WY ET AL. Quercetin suppresses proinflammatory cytokines production through MAP kinases and NF-Kappa B pathway in lipopolysaccharide-stimulated macrophage. Mol Cell Biochem 2003; 243: 153-60.

49. Subbaramaiah K, Chung WJ, Michaluart P, Telang $\mathrm{N}$, Tanabe T, Inoue H et al. Resveratrol inhibits cyclooxygenase- 2 transcription and activity in phorbol ester-treated human mammary epithelial cells. J Biol Chem 1998; 21: 21875-82.

50. Pendurthi UR, Wiwams JT, Rao VM. Resveratrol, a polyphenolic compound found in wine, inhibits tissue factor expression in vascular cells: a possible mechanism for the cardiovascular benefits associated with moderate consumption of wine. Arterioscler Thromb Vasc Biol 1999; 19: 419-26. 
51. Murase T, Kume N, Hase T, Shibuya Y, Nishizawa $Y$, ToKIMITSU I ET AL. Gallates inhibit cytokine-induced nuclear translocation of NF-KB and expression of leukocyte adhesion molecules in vascular endothelial cells. Arterioscler Thromb Vasc Biol 1999; 19: 1412-20.

52. Abou-Agag LH, Aikens ML, Tabengwa EM, Benza RL, Shows SR, GrenetT HE ET AL. Polyphenolics increase t-PA and u-PA gene transcription in cultured human endothelial cells. Alcohol Clin Exp Res 2001; 25: 155-62.

53. Parks DA, Booyse FM. Cardiovascular Protection by Alcohol and Polyphenoles. Role of Nitric Oxide. Ann NY Acad Sci 2002; 957: 115-21.

54. Padilla E, Ruiz E, Redondo S, Gordillo-Moscoso A, Slowing K, Tejerina T. Relationship between vasodilation capacity and phenolic content of Spanish wines. Eur J Pharmacol 2005; 517: 84-91.
55. Grenett HE, Abou-Agag LH, Tresnak JK, Tabengwa EM, PARKs DA, Booyse FM. Ethanol and polyphenolics rapidly decrease in vivo PAI-1 mRNA expression in rat aortic endothelium. Alcohol Clin Exp Res 2000; 24: 128A.

56. Dong Z, Ma W, Huang C, Yang CS. Inhibition of tumor promoter-induced activator protein 1 activation and cell transformation by tea polyphenols (-) epigallocatechin gallate, and theaflavins. Cancer Res 1997; 1: 4414-9.

57. Kobuchi H, Roy S, Sen CK, Nguyen HG, Packer L. Quercetin inhibits inducible ICAM-1 expression in human endothelial cells through the JNK pathway. Am J Physiol 1999; 277: C403-11.

58. Song L, Xu M, Lopes-Virella MF, Huang Y. Quercetin inhibits matrix metalloproteinase-1 expression in human vascular endothelial cells through extracellular signal-regulated kinase. Arch Biochem Biphys 2000; 39: 72-8. 